\title{
Modelling stellar coronal magnetic fields
}

\author{
Moira Jardine $^{1}$, Jean-Francois Donati ${ }^{2}$, Doris Arzoumanian ${ }^{1}$ \\ and Aline de Vidotto ${ }^{1}$ \\ ${ }^{1}$ SUPA, School of Physics and Astronomy, University of St Andrews, North Haugh, \\ StAndrews, KY16 9SS, UK \\ email:mmj@st-andrews.ac.uk, Aline.Vidotto@st-andrews.ac.uk,doris.arzoumanian@cea.fr \\ ${ }^{2}$ LATT, CNRS-UMR 5572, Obs. Midi-Pyrénées, 14 Av. E. Belin, F-31400 Toulouse, France \\ email: donati@ast.obs-mip.fr
}

\begin{abstract}
Our understanding of the structure and dynamics of stellar coronae has changed dramatically with the availability of surface maps of both star spots and also magnetic field vectors. Magnetic field extrapolations from these surface maps reveal surprising coronal structures for stars whose masses and hence internal structures and dynamo modes may be very different from that of the Sun. Crucial factors are the fraction of open magnetic flux (which determines the spin-down rate for the star as it ages) and the location and plasma density of closed-field regions, which determine the X-ray and radio emission properties. There has been recent progress in modelling stellar coronae, in particular the relative contributions of the field detected in the bright surface regions and the field that may be hidden in the dark star spots. For the Sun, the relationship between the field in the spots and the large scale field is well studied over the solar cycle. It appears, however, that other stars can show a very different relationship.
\end{abstract}

Keywords. Stars:magnetic fields, stars:coronae, stars:imaging, stars:spots

\section{Introduction}

Just as the surface distributions of spots on stars can vary considerably between different types of stars, so too can the structure of their coronae. For massive stars, the nature of the coronal magnetic field may have an influence on the structure of the wind (which can remove a significant fraction of the star's mass over its lifetime). We do not, however, know if the magnetic fields of these stars are generated by dynamo processes (Charbonneau \& MacGregor 2001; Brun et al. 2005; Spruit 2002; Tout \& Pringle 1995; MacDonald \& Mullan 2004; Mullan \& MacDonald 2005; Maeder \& Meynet 2005) or if they are fossil fields (Moss 2001; Braithwaite \& Spruit 2004; Braithwaite \& Nordlund 2006). An equally challenging question is the source and generation mechanism of the observed X-ray emission (Ignace et al. 2010).

In the case of solar-mass stars, the nature of the magnetic cycles and the lifetimes of active regions are topics that will benefit enormously from the data that will soon be available from CoRoT and Kepler. As has been mention already in this symposium, the variation with mass of the differential rotation is another crucial question. At the bottom end of the main sequence, the low mass, fully convective stars appear to have much simpler, stronger fields than their higher-mass counterparts. While a decade or so ago, it was believed that these stars could only generate small-scale magnetic fields (Durney et al. 1993; Cattaneo 1999), more recent studies have suggested that large scale fields may be generated. These models differ, however, in their predictions for the form of this field and the associated latitudinal differential rotation. They predict that the fields should be either axisymmetric with pronounced differential rotation (Dobler et al. 2006), nonaxisymmtric with minimal differential rotation (Küker \& Rüdiger 1997, 1999; Chabrier 

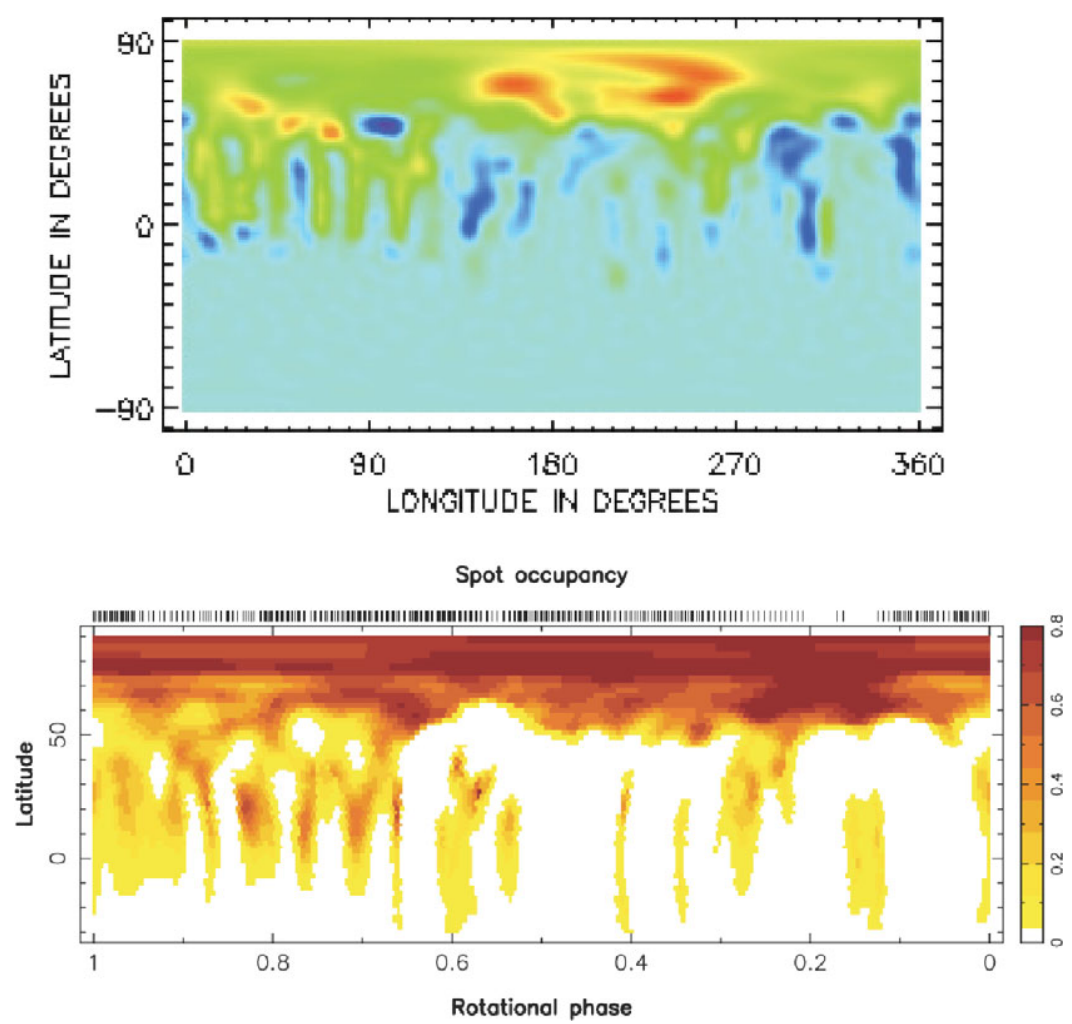

Figure 1. Zeeman-Doppler maps of the radial field component (top) and the corresponding spot occupancy map (bottom) for the AB Dor (observations carried out in 2004).

\& Küker 2006). More recently, however, Browning (2008) has published a dynamo model that produces a highly symmetric field with little differential rotation. This field structure may have implications for the nature of the spin-down of these low mass stars, since they show puzzlingly-high rotation rates, even on the main sequence.

Even in the pre-main sequence phase, the structure of star's corona can be important since it affects the rate at which the star can accrete mass from its disk (Gregory et al. 2006b, 2010). Low mass stars remain fully convective throughout their evolution onto the main sequence, but higher mass stars will develop a radiative core at some stage perhaps even while they are still accreting from their disks. This difference in internal structure may lead to different dynamo behaviours, and indeed there is a suggestion from observations of a handful of stars that the field structures of stars with a radiative core (and hence a tachocline where a solar-like interface dynamo might be active) are more complex than those of the fully-convective stars (Donati et al. 2007, 2008b; Jardine et al. 2008; Gregory et al. 2008; Hussain et al. 2009). The implications for the spin evolution of the stars have not yet been fully explored however.

\section{Modelling stellar coronae}

The first step in modelling the corona of a star is to determine the form of the surface field. This is most commonly done using the technique of Zeeman-Doppler imaging (Donati \& Collier Cameron 1997; Donati et al. 1999). This typically shows a complex 

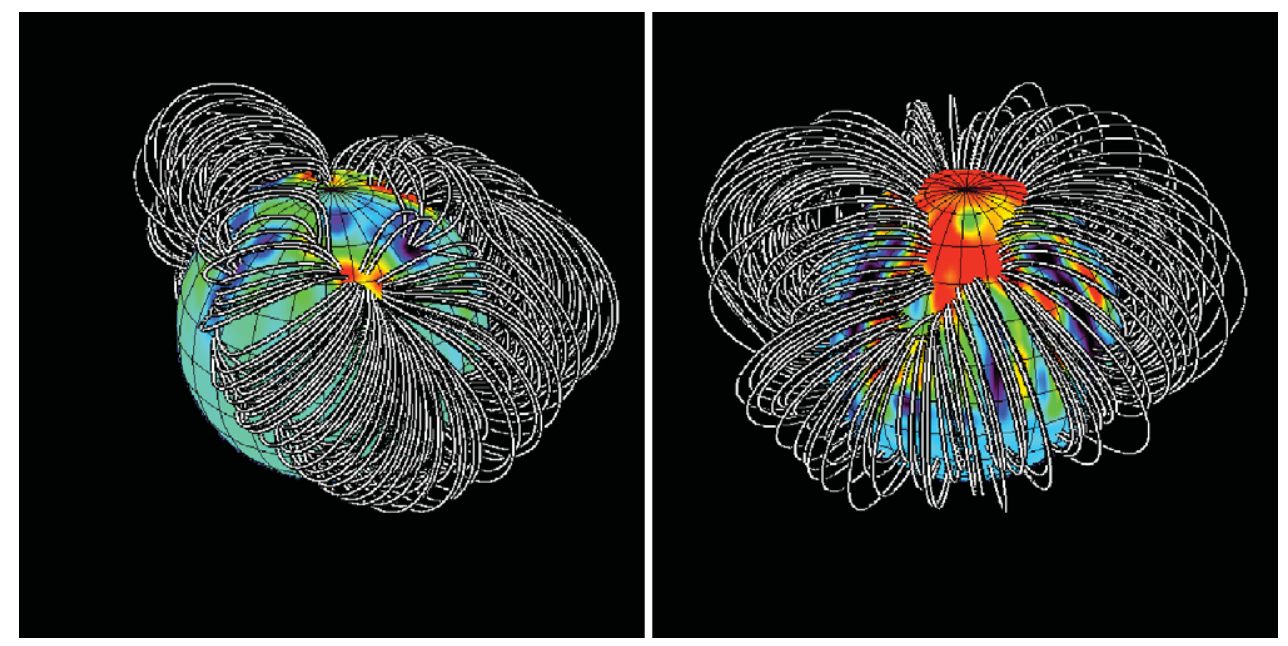

Figure 2. Field extrapolation from the original Zeeman-Doppler map shown in Fig. 1 (left) and from the maps of the combined Zeeman-Doppler and spot field (right).

distribution of surface spots that is often very different from that of the Sun, with spots and mixed polarity flux elements extending over all latitudes up to the pole. A comprehensive review of starspot distributions is given in Strassmeier (2009).

While the methods of extrapolating the magnetic field from these magnetograms may differ, the basic procedure is the same. The most commonly-used method is the Potential Field Source Surface method (Altschuler \& Newkirk, Jr. 1969; Jardine et al. 1999; Jardine et al. 2001; Jardine et al. 2002a; McIvor et al. 2003), but it is also possible to use nonpotential fields (Donati 2001; Hussain et al. 2002). Once the 3D structure of the magnetic field has been determined, the structure of the coronal gas can be determined by assuming that the gas trapped on these field lines is in isothermal, hydrostatic equilibrium. We can then determine the coronal gas pressure, subject to an assumption for the gas pressure at the base of the corona. We assume that it is proportional to the magnetic pressure, i.e. $p_{0} \propto B_{0}^{2}$, where the constant of proportionality is determined by comparison with X-ray emission measures (Jardine et al. 2002b; Jardine et al. 2006; Gregory et al. 2006a). For an optically thin coronal plasma, this then allows us to produce images of the X-ray emission.

\section{Effect of spot magnetic fields on coronal structure}

The fraction of a star's surface that is covered in spots and the strength and degree of complexity of the coronal magnetic field all vary with stellar mass. While low mass, fully convective stars commonly have strong, simple surface fields with few spots (Donati et al. 2008a; Morin et al. 2008, 2010), solar mass stars such as AB Dor (shown in Fig 1) typically have complex fields and spots that can cover a significant fraction of their surfaces (Strassmeier 2009). Zeeman-Doppler imaging does not recover all of the magnetic field in these spotted regions - a factor that may be more important in stars with greater spot coverage (Johnstone et al. 2010). Although we do no know the polarity of the magnetic field in the spots, we can take a statistical approach. We randomly allocate a polarity to each spot (defined at a certain level of spot occupancy) and then add this field to the surface field reconstructed by Zeeman-Doppler imaging. By repeating this process many times and each time extrapolating the coronal field, we can create 


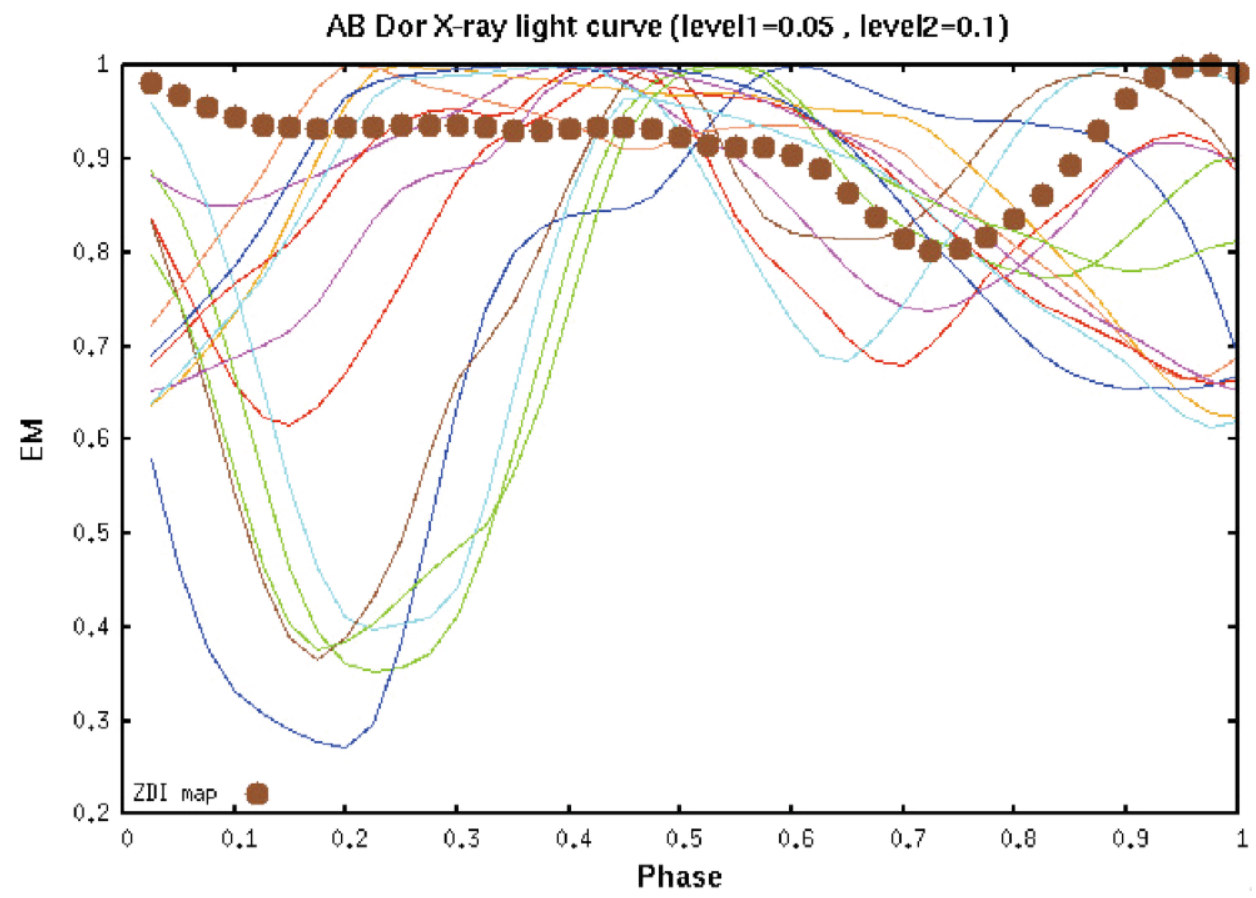

Figure 3. X-ray rotational modulation of $\mathrm{AB}$ Dor. The dots represent the X-ray rotational modulation calculated from the ZDI map. The lines represent the X-ray rotational modulation using the combined maps (ZDI + spot maps).

many realisations of the structure of the corona (see for example Figs 2). By doing this for stars of different masses, we can determine the possible effect that the flux hidden in the spots might have on the corona (Arzoumanian et al. 2010). Because the spot field typically has a greater degree of axial symmetry, this process often shifts the large-scale dipole axis closer to the rotation axis. The fraction of open flux and the magnitude of the X-ray emission measure also typically increase. Fig. 3 shows some examples of the $\mathrm{X}$-ray light curves that might be produced.

\section{Moving towards full MHD}

While the potential field source surface models have the advantage of computational speed and simplicity, they only allow indirectly for the effect of the stellar wind. In order to improve our understanding of the interplay between coronal and wind processes in stars we need to incorporate the observed surface magnetograms into an MHD code (Cohen et al. 2010; Vidotto et al. 2010). First results from this for the fully-convective star V374 Peg have been extremely interesting (see Fig. 4). Like many low mass stars, V374 Peg rotates extremely rapidly and has a strong, almost dipolar field. This means that magnetic forces dominate over the other forces and results in a very non-solar, magnetocentrifugally driven wind. The general scalings that emerge from this study are that both the angular momentum loss rate and the mass loss rate scale as $n^{1 / 2}$ (where $n$ is the density) and hence the spin-down time scales as $\mathrm{n}^{-1 / 2}$. The fact that V374 Peg is a rapid rotator therefore suggests it has a coronal density that is low by stellar standards, if high relative to the Sun. The mass loss rate and speed of the wind are both also higher than 


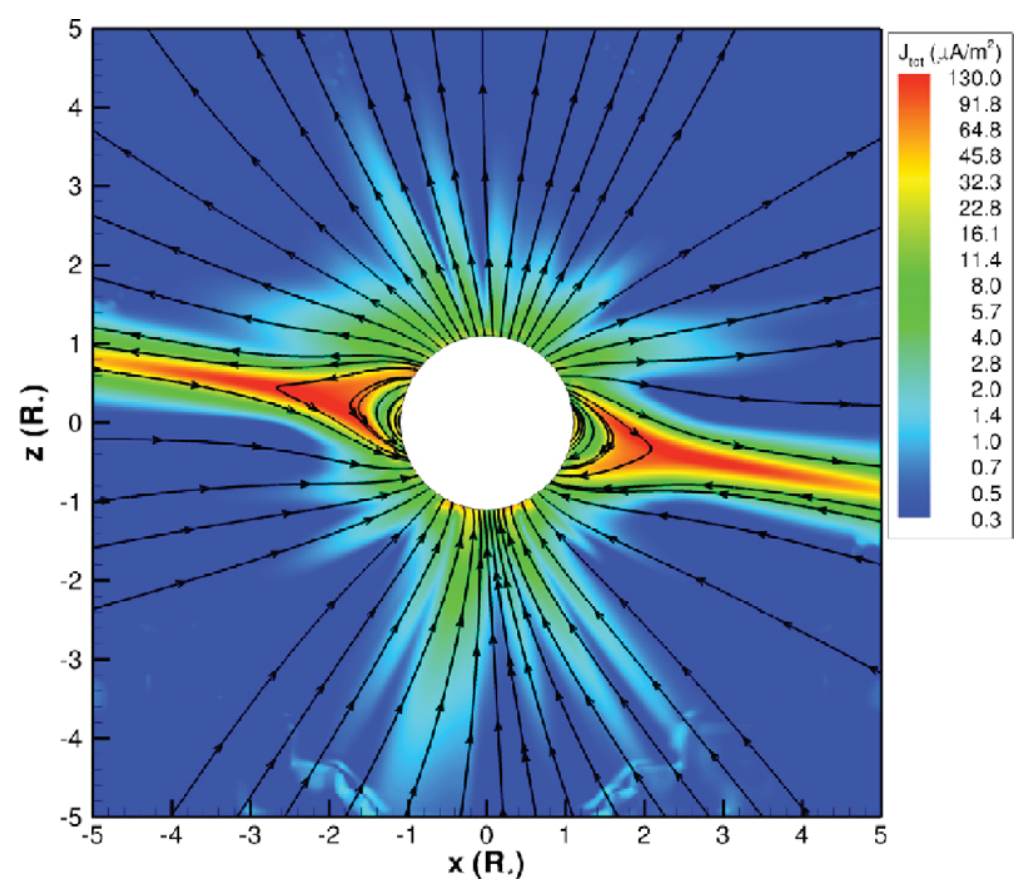

Figure 4. Current density.

solar values. Most interesting, however, is the ram pressure which may be five orders of magnitude higher than that of the solar wind. This has significant implications for planet habitability, since such a high ram pressure may crush planetary magnetospheres. If, for example, we place a planet in the habitable zone of an M-dwarf star, it needs a field of about $8 \mathrm{G}$ (around half that of Jupiter) to maintain a magnetosphere. The means by which such a field might be generated in the planetary interior are still a matter of debate.

\section{Conclusions}

The availability of spot maps and magnetograms for stars other than the Sun has revolutionised our view of stellar magnetic fields. By using these maps to model the coronal structure of a range of stars we can also gain insight into their winds, their rotational evolution and their coronal emission. Advances in MHD modelling that incorporate the three vector components of the surface magnetic field promise to bring about a similar revolution.

\section{References}

Altschuler, M. D. \& Newkirk, Jr., G. 1969, Solar Phys., 9, 131

Arzoumanian, D., Jardine, M., Donati, J., Morin, J., \& Johnstone, C. 2010, ArXiv e-prints Braithwaite, J. \& Nordlund, A. 2006, Astron. Astrophys, 450, 1077

Braithwaite, J. \& Spruit, H. C. 2004, Nature, 431, 819

Browning, M. K. 2008, Astrophys. J., 676, 1262

Brun, A. S., Browning, M. K., \& Toomre, J. 2005, Astrophys. J., 629, 461

Cattaneo, F. 1999, Astrophys. J., 515, L39 
Chabrier, G. \& Küker, M. 2006, Astron. Astrophys, 446, 1027

Charbonneau, P. \& MacGregor, K. B. 2001, Astrophys. J., 559, 1094

Cohen, O., Drake, J. J., Kashyap, V. L., Hussain, G. A. J., \& Gombosi, T. I. 2010, ArXiv e-prints

Dobler, W., Stix, M., \& Brandenburg, A. 2006, Astrophys. J., 638, 336

Donati, J., Morin, J., Petit, P., et al. 2008a, Mon. Not. Roy. Astron. Soc., 390, 545

Donati, J.-F. 2001, LNP Vol. 573: Astrotomography, Indirect Imaging Methods in Observational Astronomy, 573, 207

Donati, J.-F. \& Collier Cameron, A. 1997, Mon. Not. Roy. Astron. Soc., 291, 1

Donati, J.-F., Collier Cameron, A., Hussain, G., \& Semel, M. 1999, Mon. Not. Roy. Astron. Soc., 302, 437

Donati, J.-F., Jardine, M. M., Gregory, S. G., et al. 2007, Mon. Not. Roy. Astron. Soc., 380, 1297

Donati, J.-F., Jardine, M. M., Gregory, S. G., et al. 2008b, Mon. Not. Roy. Astron. Soc., 386, 1234

Durney, B. R., De Young, D. S., \& Roxburgh, I. W. 1993, Solar Phys., 145, 207

Gregory, S. G., Jardine, M., Cameron, A. C., \& Donati, J.-F. 2006a, Mon. Not. Roy. Astron. Soc., 373,827

Gregory, S. G., Jardine, M., Gray, C. G., \& Donati, J. 2010, ArXiv e-prints

Gregory, S. G., Jardine, M., Simpson, I., \& Donati, J.-F. 2006b,Mon. Not. Roy. Astron. Soc., 371,999

Gregory, S. G., Matt, S. P., Donati, J., \& Jardine, M. 2008, Mon. Not. Roy. Astron. Soc., 389, 1839

Hussain, G. A. J., Collier Cameron, A., Jardine, M. M., et al. 2009, Mon. Not. Roy. Astron. Soc., 398, 189

Hussain, G. A. J., van Ballegooijen, A. A., Jardine, M., \& Collier Cameron, A. 2002, Astrophys. $J ., 575,1078$

Ignace, R., Oskinova, L. M., Jardine, M., et al. 2010, ArXiv e-prints

Jardine, M., Barnes, J., Donati, J.-F., \& Collier Cameron, A. 1999, Mon. Not. Roy. Astron. Soc., 305, L35

Jardine, M., Collier Cameron, A., \& Donati, J.-F. 2002a, Mon. Not. Roy. Astron. Soc., 333, 339

Jardine, M., Collier Cameron, A., Donati, J.-F., Gregory, S. G., \& Wood, K. 2006, Mon. Not. Roy. Astron. Soc., 367, 917

Jardine, M., Collier Cameron, A., Donati, J.-F., \& Pointer, G. 2001, MNRAS, 324, 201

Jardine, M., Wood, K., Collier Cameron, A., Donati, J.-F., \& Mackay, D. H. 2002b, Mon. Not. Roy. Astron. Soc., 336, 1364

Jardine, M. M., Gregory, S. G., \& Donati, J. 2008, Mon. Not. Roy. Astron. Soc., 386, 688

Johnstone, C., Jardine, M., \& Mackay, D. H. 2010, Mon. Not. Roy. Astron. Soc., 404, 101

Küker, M. \& Rüdiger, G. 1997, Astron. Astrophys, 328, 253

Küker, M. \& Rüdiger, G. 1999, in ASP Conf. Ser. 178: Workshop on stellar dynamos, Vol. 178, $87-96$

MacDonald, J. \& Mullan, D. J. 2004, Mon. Not. Roy. Astron. Soc., 348, 702

Maeder, A. \& Meynet, G. 2005, Astron. Astrophys, 440, 1041

McIvor, T., Jardine, M., Cameron, A. C., Wood, K., \& Donati, J.-F. 2003, Mon. Not. Roy. Astron. Soc., 345, 601

Morin, J., Donati, J., Petit, P., et al. 2008, Mon. Not. Roy. Astron. Soc., 390, 567

Morin, J., Donati, J., Petit, P., et al. 2010, Mon. Not. Roy. Astron. Soc., 1077

Moss, D. 2001, in ASP Conference Series, Vol. 248, Magnetic fields across the HertzsprungRussell diagram, ed. S. G. Mathys \& D. Wickramasinghe (San Francisco), 305

Mullan, D. J. \& MacDonald, J. 2005, Mon. Not. Roy. Astron. Soc., 356, 1139

Spruit, H. 2002, Astron. Astrophys, 381, 923

Strassmeier, K. G. 2009, A. Ast. Rev., 17, 251

Tout, C. A. \& Pringle, J. E. 1995, Mon. Not. Roy. Astron. Soc., 272, 528

Vidotto, A., Jardine, M., Opher, M., Donati, J.-F., \& Gombosi, T. I. 2010, Mon. Not. Roy. Astron. Soc. (in press) 


\section{Discussion}

STRASSMEIER: You are quite right with our inability to reconstruct magnetic field from flux deficient spots. However, we know that this is an artifact and recent results by Berdyugina and her group showed the existence of fields in starspots.

JARDINE: I think it would, and I think the solar community should solve the problem for the sun as well. It's - it's only true in its very [INAUDIBLE] limit where you have the very, very strong fields. It's not true for a solar type. So it's specific to these low mass stars in their incredibly strong field and rapid rotation. We tried several different types of assumption. We tried two types of star. We took Capella and V374Peg which has very small thin red pots, and we tried three different ways of allocating the field to the spot - so taking a different brightness contour to be a different field strength. The results you get depend on what you assume. I think it would be very nice actually to have some input perhaps from the solar community as to what might be a better way to do that. We recently tried three different parametration.

DUPREE: I have a wind question. If the sun was rapidly rotating and the centrifugally driven wind, how would the profile of the wind compare as to our standard solar wind?

JARDINE: It would be very much faster, and the RAM pressure would be very much greater. The critical point - the critical point essentially depends on the temperature. So it would depend on how hot the wind is. If the sun was still at its same temperature, the critical point would be the at the same point. So the sonic point would be the same; but the terminal velocity would be very different, much higher. 